\title{
Strong thermomechanical squeezing in a far-detuned membrane-in-the-middle system
}

\author{
Sameer Sonar, ${ }^{1,2,{ }^{*}}$ Vitaly Fedoseev, ${ }^{2}$ Matthew J. Weaver, ${ }^{3}$ Fernando Luna, ${ }^{3}$ Elger Vlieg, ${ }^{2}$ Harmen van der Meer, ${ }^{2}$ \\ Dirk Bouwmeester, ${ }^{3,2}$ and Wolfgang Löffler ${ }^{2, \dagger}$ \\ ${ }^{1}$ Department of Physics, Indian Institute of Technology-Bombay, Powai, Mumbai 400076, India \\ ${ }^{2}$ Huygens-Kamerlingh Onnes Laboratorium, Universiteit Leiden, 2333 CA Leiden, The Netherlands \\ ${ }^{3}$ Department of Physics, University of California, Santa Barbara, California 93106, USA
}

(Received 24 May 2018; published 3 July 2018)

\begin{abstract}
We demonstrate $8.5 \mathrm{~dB}$ thermal squeezing of a membrane oscillator using the dynamical backaction effect and electrostatic feedback in an optomechanical membrane-in-the-middle setup. We show that strong squeezing can be obtained even in the far-detuning regime of a sideband-resolved system. By using the dielectrophoretic force of a metallic needle kept in close proximity to the membrane, we implement the one-quadrature active feedback scheme to prevent the divergence of the amplified quadrature and surpass the $3 \mathrm{~dB}$ limit of mechanical squeezing. We also discuss different regions of the sideband spectrum where strong squeezing can be obtained. Although the demonstration here is classical, this technique is equally applicable to prepare the mechanical oscillator in a quantum squeezed state.
\end{abstract}

DOI: 10.1103/PhysRevA.98.013804

\section{INTRODUCTION}

Methods to prepare a mechanical oscillator in a squeezed state [1] are important to enhance the read-out sensitivity and to reduce the measurement backaction in the quadrature of interest. Here we discuss quadrature-squeezed states where the noise in the $\sin \omega t$ and $\cos \omega t$ quadratures are not equal, in contrast to coherent and thermal states. Several methods have been proposed to generate squeezed mechanical states both in the classical [1,2] and the quantum [3-7] regimes. In the quantum regime, quantum backaction evading measurements have demonstrated noise reduction close to the zero-point motion [8] and even beyond [9-13].

Classical squeezed mechanical states or thermally squeezed states have been generated in opto- or electromechanical systems by applying a parametric force [1,14-18], optically modulating the spring constant [19], fast switching between two trapped frequencies [20], and quantum nondemolition measurements [21]. Further, it is also possible to generate squeezed states involving a quadrature of two different mechanical oscillators [22-25].

The thermal excitation of a mechanical oscillator leads to a Gaussian noise distribution, where the noise amplitudes in both quadratures are equal. Parametric modulation of the spring constant at twice the mechanical frequency breaks this degeneracy of the two quadratures [1]. The thermal noise in the quadrature which is in-phase with this modulation increases, while the thermal noise in the orthogonal out-ofphase quadrature decreases. When the noise amplitude in one quadrature is reduced by a factor of $3 \mathrm{~dB}$, the orthogonal one becomes infinitely large. Above the $3 \mathrm{~dB}$ limit, the system behavior becomes chaotic due to the parametric instability

\footnotetext{
*sameersonar@iitb.ac.in

†loeffler@physics.leidenuniv.nl
}

in the diverging quadrature [14]. Fortunately, it is possible to prevent the divergence and the parametric instability and enable squeezing of the orthogonal quadrature beyond the 3 $\mathrm{dB}$ limit. Experiments performed to surpass this limit include active feedback on the diverging quadrature [15,18], real-time squeezing phase adjustment [17], detuned pump [16], and reservoir engineering [13].

The radiation force acting on a mechanical oscillator in an optomechanical system has the important property that its response to a change in the oscillator position is delayed by approximately the cavity decay time. This delay modifies the damping and the frequency of the mechanical oscillator as a function of the laser detuning and is called the dynamical backaction effect [26]. In a sideband-unresolved system where the mechanical frequency $\Omega_{m}$ is smaller than the cavity decay rate $\kappa$, the dynamical backaction is significant only if the laser is near-resonance with the optical cavity mode. To our knowledge, this is the only regime of the sideband spectrum in which optomechanical squeezing beyond $3 \mathrm{~dB}$ has been demonstrated [19]. On the other hand, in the sideband-resolved regime $\left(\Omega_{m}>\kappa\right)$, sideband cooling can be used to cool the mechanical oscillator to its quantum ground state [27]. Also, using a far-detuned laser is important in experiments where one wants to perform a squeezing operation on an arbitrary initial state without any additional heating or cooling effects.

Here, we show optomechanical squeezing for a well sideband-resolved optomechanical system with $\Omega_{m} / \kappa \approx 9$, where the dynamical backaction is efficient even in the fardetuned regime. We obtain $8.5 \mathrm{~dB}$ mechanical squeezing using the dynamical backaction effect at $1.43 \times \Omega_{m}$ laser detuning. This strong squeezing despite large detuning is also facilitated by using a mechanical oscillator with a reasonably high $Q$ factor of $3 \times 10^{5}$ at room temperature that enables higher spring constant modulation [15] and consequently more squeezing. 
We first present our membrane-in-the-middle setup and discuss parametric squeezing for different laser detunings. Then we show $3 \mathrm{~dB}$ squeezing accompanied by the parametric instability in one quadrature. Finally, we demonstrate how to surpass the $3 \mathrm{~dB}$ limit by applying feedback onto the diverging quadrature of the mechanical oscillator.

\section{EXPERIMENT}

Our optomechanical system consists of a $98 \mathrm{~mm}$ FabryPerot cavity operating at $1064 \mathrm{~nm}$. High-quality thin-film mirrors consisting of alternating $\mathrm{Ta}_{2} \mathrm{O}_{5} / \mathrm{SiO}_{2}$ layers are mounted at the end of a $98 \mathrm{~mm}$ Invar spacer tube similar to [28]. The radius of curvature of the mirrors is $5 \mathrm{~cm}$, which gives strong near-critical focusing at the center of the cavity. The setup is placed inside a vacuum chamber at a pressure of $10^{-6}$ mbar.

The mechanical oscillator used in this scheme is a high-stress $50 \mathrm{~nm}$ silicon nitride semitransparent membrane (NORCADA Inc.). The fundamental mode has a resonance frequency $\Omega_{m} / 2 \pi \approx 385 \mathrm{kHz}$, effective mass $m_{\mathrm{eff}} \approx 33 \mathrm{ng}$, and a $Q$ factor of $3 \times 10^{5}$ at room temperature. The membrane is placed in the middle of the cavity, and its tilt and axial position are controlled using 3 piezo motors. The axial position of the membrane with respect to the standing-wave pattern inside the cavity is chosen to give large first-order singlephoton optomechanical coupling $g_{0}$ [29]. We obtain an optical finesse of the fundamental (Gaussian) cavity mode of 33000 , and a membrane coupling of $g_{0} / 2 \pi=3 \mathrm{~Hz}$ from calculating the derivative of the dispersion curve.

The optical setup is shown in Fig. 1. We use two lasers: The probe laser $(\approx 10 \mu \mathrm{W})$ is used for sensitive readout of the motion of the membrane. We use the Pound-Drever-Hall (PDH) method [30] to lock the laser to the cavity. The PDH error signal is demodulated at the mechanical frequency and is used to observe the real-time phase-space trajectory of the fundamental mechanical mode: $x(t)=X_{1}(t) \sin \left(\Omega_{m} t\right)+$ $X_{2}(t) \cos \left(\Omega_{m} t\right)$. A second, more powerful $(\approx 400 \mu \mathrm{W})$ pump

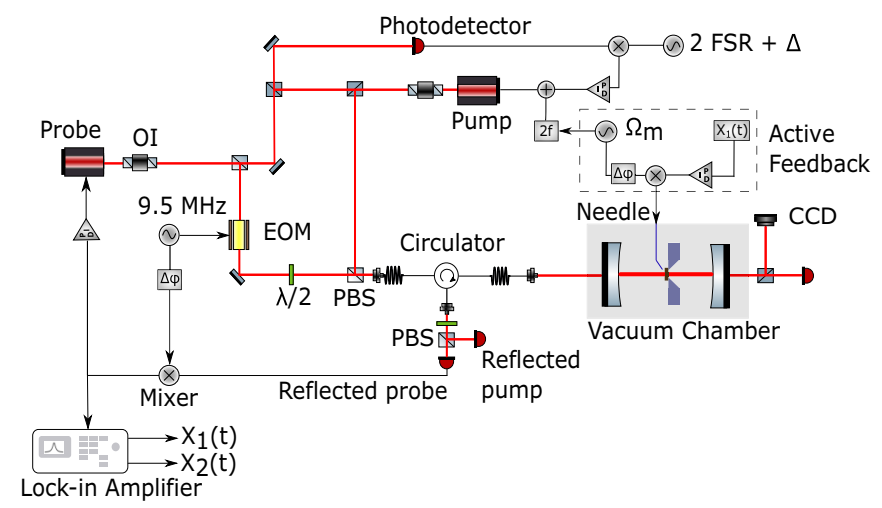

FIG. 1. Experimental membrane-in-the-middle setup. The probe laser is locked to the optical cavity resonance frequency with the Pound-Drever-Hall (PDH) technique and used to record the motion of the membrane, the pump laser applies the parametric force, and the needle generates the parametric electrophoretic force. EOM: electrooptical modulator; OI: optical isolator; PID: proportional-integral differential feedback controller; PBS: polarizing beam splitter; CCD: charge coupled device. laser is used to impart radiation force onto the membrane. This laser is locked to the probe laser with an optical phaselocked loop and a 2 free spectral range (FSR) offset to avoid any unwanted interference. Using an offset of 2 FSR is advantageous over 1 FSR because unavoidable nm-scale drifts of the membrane position lead (ideally) to no changes in the frequency spacing of cavity resonances separated by 2 FSR. [28]. The other means to influence the motion of the membrane is the dielectrophoretic force of a metal needle situated near the membrane [31]. The needle is far enough from the center of the membrane that it does not interfere with the optical beam but is close enough to create a large electric-field gradient.

We follow the analysis given in [15] for parametric driving, taking into account the additional optical damping $\Gamma_{\text {opt }}$. The equation for a squeezed thermomechanical oscillator is given by

$$
\ddot{x}+\Gamma_{\text {eff }} \dot{x}+\Omega_{m}^{2}\left[1-\frac{2 g}{Q^{\prime}} \sin \left(2 \Omega_{m} t+\phi\right)\right] x=F_{\text {th }}(t) .
$$

Here, $\Gamma_{\text {eff }}=\Gamma_{m}+\Gamma_{\text {opt }}$ is the effective damping, $Q^{\prime}=$ $\Omega_{m} / \Gamma_{\text {eff }}$ is the effective $Q$ factor, $F_{\text {th }}(t)$ is the thermal Langevin force which is responsible for the Brownian motion, and $g$ is the gain parameter of the spring constant modulation. Since this modulation is exactly at twice the mechanical frequency, the two quadratures experience different forces. The one which is in phase with this modulation is amplified in variance and the orthogonal one is squeezed. For $\phi=0$, the variance of each quadrature, $X_{1}$ and $X_{2}$, is given by

$$
\left\langle X_{1}^{2}\right\rangle=\frac{k_{B} T_{\mathrm{eff}}}{m_{\mathrm{eff}} \Omega_{m}^{2}} \frac{1}{(1-g)},\left\langle X_{2}^{2}\right\rangle=\frac{k_{B} T_{\mathrm{eff}}}{m_{\mathrm{eff}} \Omega_{m}^{2}} \frac{1}{(1+g)} .
$$

Here, $k_{B}$ is the Boltzmann constant and $T_{\text {eff }}$ is the effective temperature of the fundamental mode which takes into account the optical damping. Changing the modulation phase by $\phi$ changes the squeezing direction by $\phi / 2$ [17]. We change this offset to align the squeezing axis with the $X_{2}$ quadrature. Figure 2 shows the measurement of the effective damping $\Gamma_{\text {eff }}$ and the mechanical frequency shift $\delta \Omega_{m}$ as a function of pump

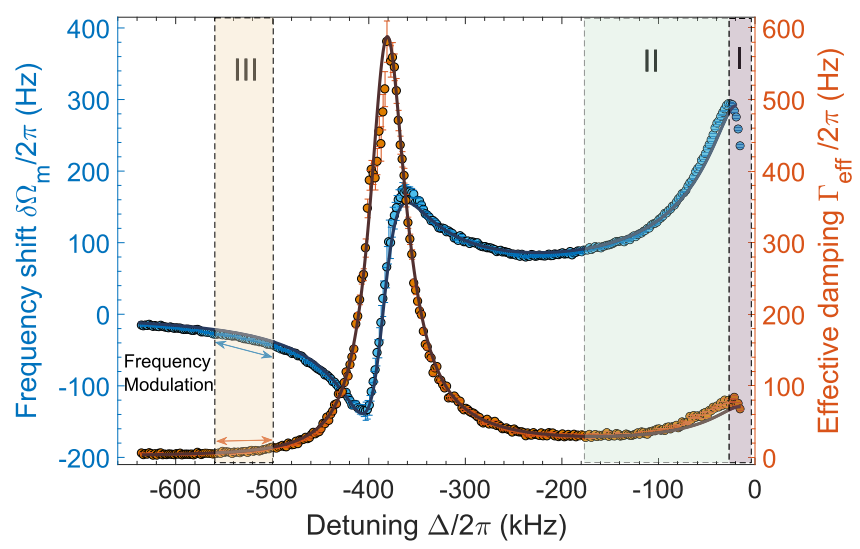

FIG. 2. Measurement of the effective damping $\Gamma_{\text {eff }}$ (red) and mechanical frequency shift $\delta \Omega_{m}$ (blue) depending on the pump laser detuning from the cavity resonance (at $\Delta=0$ ). Regions I-III show potential regions to perform spring constant modulation by frequency modulation of the laser. The solid lines represent theoretical fits to the experimental data. 
laser detuning $\Delta$. We place a laser beam in the shaded region III which is $1.43 \times \Omega_{m}$ detuned on the red side and perform frequency modulation. The nonzero slope of the frequencyshift curve leads to modulation of the spring constant. We note that the frequency spectrum of our membrane is slightly anharmonic and the higher-order modes deviate slightly from their expected values by few hundred Hertz, such that the frequency of the $(2,2)$ mode is not exactly twice that of the $(1,1)$ mode. This deviation works in our favor because driving the membrane at twice the mechanical frequency of the $(1,1)$ mode does not excite the $(2,2)$ mode. The feedback bandwidth of the optical phase-locked loop is much smaller than $\Omega_{m}$ and hence the frequency modulation at $2 \Omega_{m}$ does not affect the optical phase-locked loop. In the shaded region, the change in frequency shift is 2.7 times higher than the change in effective damping and we treat the effective damping as a constant to simplify the analysis. This approximation is not valid, for example, near the red sideband where the effective damping is highly nonlinear and completely overwhelms the frequency shift. In this case, one also needs to consider the modulation of the effective damping in Eq. (1), which leads to cross coupling between the two quadratures and the variances in the two quadratures cannot be written in a decoupled way as in Eq. (2).

Figure 2 also shows other regions of the sideband spectrum where the derivative of the frequency-shift curve $\delta \Omega_{\mathrm{m}}$ is higher than that of the effective damping $\Gamma_{\text {eff }}$ and where strong squeezing can be obtained. For instance, the study in [19] operates in region I close to zero detuning. Our sideband-resolved system is unstable in this region because the mechanical oscillator gets driven as soon as the pump beam is slightly detuned on the blue side, making this region impractical to use. Additionally, regions I and II have a larger second-order derivative of the frequency shift and effective damping curves, making the spring constant modulation in Eq. (1) "nonlinear." We select region III to maximally decouple optical damping from squeezing.

Figure 3(a) shows the phase-space thermal distribution of the resonator for the case when the pump laser is at $\Delta=$ $1.43 \times \Omega_{m}$ on the red side (in region III). The resulting optical damping leads to an effective temperature $T_{\text {eff }}=120 \mathrm{~K}$. The data are recorded for 5 seconds with a demodulation bandwidth of $138 \mathrm{~Hz}$. The distribution is Gaussian in both quadratures with equal variances. Applying $2 \Omega_{m}$ frequency modulation to the laser leads to a squeezed distribution, as shown in Fig. 3(b). Increasing the gain parameter $g$ slightly above 1 leads to the parametric instability whereby the thermal distribution gets shifted from the origin. The amplified $X_{1}$ quadrature shows bistability because the equation of motion is invariant under a $\pi$ phase shift [14], while the distribution in $X_{2}$ is still squeezed. Figures 3(c) and 3(d) show this behavior as a function of time and in phase space, respectively.

\section{PARAMETRIC FEEDBACK}

The $3 \mathrm{~dB}$ limit imposed by the parametric squeezing is not a fundamental limit and can be surpassed by preventing the divergence of the amplified quadrature. We use the onequadrature active feedback technique [15] to sense the motion in the amplified quadrature and apply a force to counteract that motion. For this purpose, we use a sharp-tip metal needle
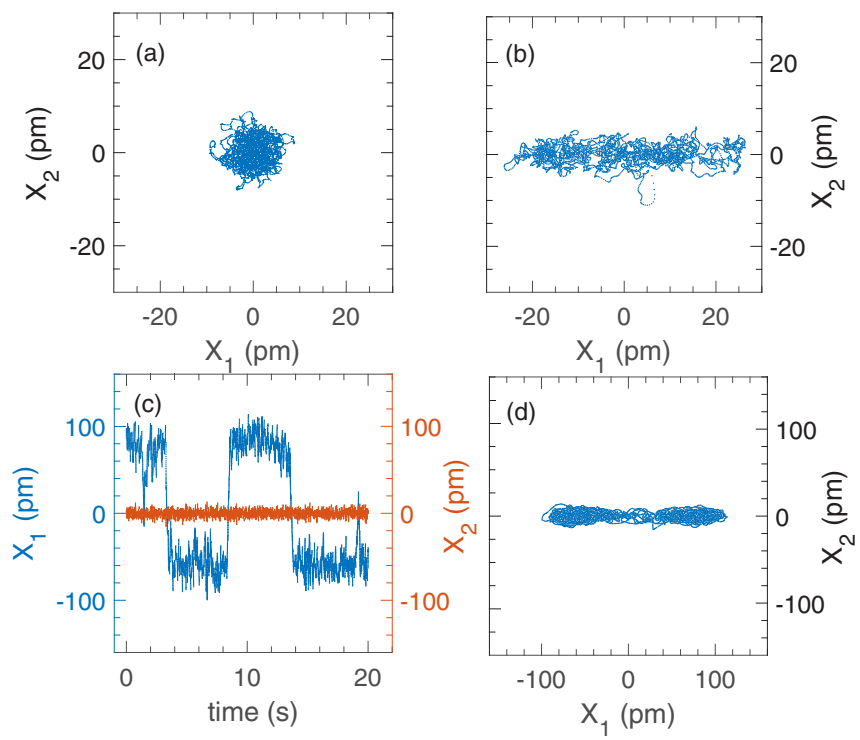

FIG. 3. (a) Phase-space trajectory of the mechanical oscillator with an effective temperature $T_{\text {eff }}=120 \mathrm{~K}$. (b) Squeezed trajectory with gain parameter $g=0.96$ after aligning the squeezing direction with $X_{2}$ quadrature. (c),(d) Bistability in the $X_{1}$ quadrature and squeezed distribution in the $X_{2}$ quadrature for $g>1$, (c) as a function of time and $(d)$ in phase space.

close to the membrane to generate a dielectrophoretic force. The feedback force added to Eq. (1) and the modified variances in the two quadrature are now given by

$$
\begin{gathered}
F_{\mathrm{fb}}(t)=-h \frac{\Omega_{m}^{2}}{Q^{\prime}} X_{1}(t) \cos \left(\Omega_{m} t\right), \\
\left\langle X_{1}^{2}\right\rangle=\frac{k_{B} T_{\mathrm{eff}}}{m_{\mathrm{eff}} \Omega_{m}^{2}} \frac{1}{(1-g+h)}, \quad\left\langle X_{2}^{2}\right\rangle=\frac{k_{B} T_{\mathrm{eff}}}{m_{\mathrm{eff}} \Omega_{m}^{2}} \frac{1}{(1+g)},
\end{gathered}
$$

where $h$ is the dimensionless feedback parameter. The feedback circuit is shown in the dashed box in Fig. 1. We mix the selected quadrature ( $X_{1}$ here), modified by appropriate PID parameters, with $\cos \Omega_{m} t$ and send it to the needle. The squeezing gain parameter $g$ of the $X_{1}$ quadrature is shifted by $h$, while the orthogonal quadrature $X_{2}$ remains independent and can now be squeezed beyond $3 \mathrm{~dB}$ because the $X_{1}$ quadrature does not diverge for $g \rightarrow 1$.

Figure 4 shows the quadrature variances for different strengths of active feedback. We normalize the variances with respect to the thermal distribution when the pump beam is $1.43 \times \Omega_{m}$ detuned on the red side. This far detuning ensures minimal sideband cooling of the initial state and allows us to perform a squeezing operation largely independent of sideband cooling. The shaded area shows the allowed parameter space without any active feedback. Increasing the feedback parameter to $h=2.9$ shifts the diverging curve (red diamonds) to the right and a maximum of $7.03 \mathrm{~dB}$ squeezing is obtained. For $h=21.5$, a lower pump power was used which resulted in an effective temperature of $182 \mathrm{~K}$. A maximum squeezing of $8.5 \mathrm{~dB}$ is obtained in this case. At large gain parameter $g$, the variance in $X_{1}$ diverges from the theoretical fit; we suspect that we reach the nonlinear region of the frequency shift and the effective damping curves outside of region III in Fig. 2. 


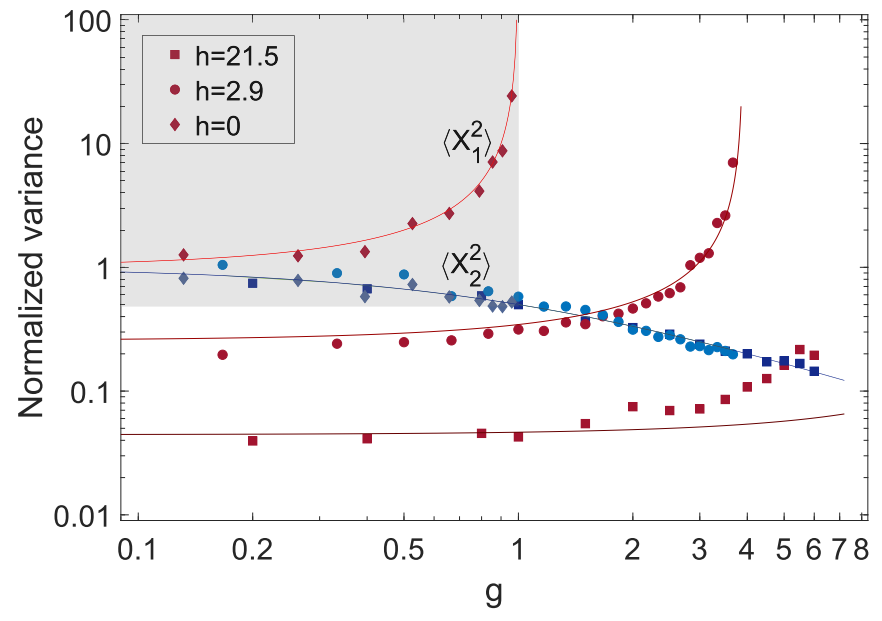

FIG. 4. Normalized variances in the $X_{1}$ and $X_{2}$ quadratures as a function of the gain parameter $g$. The shaded area highlights the allowed parameter space without any feedback. Diamonds: $h=0$; circles: $h=2.9$ and a maximum of $7.03 \mathrm{~dB}$ squeezing; squares: $h=$ 21.5 and a maximum of $8.5 \mathrm{~dB}$ squeezing. $T_{\text {eff }}$ is $120 \mathrm{~K}$ for $h=0$ and 2.9, respectively, and $182 \mathrm{~K}$ for $h=21.5$. Solid lines represent theoretical fits based on Eq. (4).

Additionally, there could be a slight mismatch between the feedback direction and the diverging quadrature [18].

\section{DISCUSSION}

In this study, we have shown that strong thermally squeezed states can be obtained using the dynamical backaction effect even in the far-detuned regime. We have implemented a simple electrical feedback scheme to surpass the $3 \mathrm{~dB}$ limit and confine the motion of the mechanical oscillator in a strongly squeezed state. By selecting the far-detuned region, we are able to minimize optomechanical cooling so that our squeezing operation can be performed on an arbitrary initial state. This method is effective to perform many quantum experiments with squeezed states such as transfer between different mechanical oscillators [32], electro-optomechanical transduction
[33], possible studies of decoherence in macroscopic objects $[34,35]$, and the enhancement of quantum synchronization [36]. Having a resolved sideband system has the advantage that there are several regions of the sideband spectrum where strong spring constant modulation can be obtained. However, the optical readout of the mechanical motion is also delayed due to the high-finesse cavity, which limits the amount of feedback cooling one can get in one quadrature.

To sum up, there are three factors that are currently limiting the amount of squeezing: the strength of the one-quadrature active feedback, the strength of the spring constant modulation, and the quality factor of the mechanical oscillator. The second issue can, in principle, be solved by using two driving tones, one on the red sideband and the other on the blue sideband, and frequency modulating both of them at twice the mechanical frequency. In this way, the damping cancels out whereas the frequency shifts due to the two tones add up, giving a large spring constant modulation. This method can also be used in the reversed dissipation regime [37], where the role of the mechanical oscillator and the optical oscillator is interchanged, to obtain strong squeezed light. Last, by using membranes with an exceptionally high-quality factor such as in [38], combined with our sideband-resolved optical cavities, it should be possible to prepare the mechanical resonator in a truly quantum squeezed state.

Recently, the key requirement of optical cooling of a membrane oscillator close to the quantum ground state was achieved [39] by using optical feedback with a $Q=10^{9}$ soft-clamped membrane in a cryostat with a base temperature of $T \sim 10 \mathrm{~K}$.

\section{ACKNOWLEDGMENTS}

This work is part of the research program of the Foundation for Fundamental Research (FOM) and of the NWO VICI research program, which are both part of the Netherlands Organization for Scientific Research (NWO). The authors would like to thank Kier Heeck for technical assistance. The authors are also grateful for useful discussions with Frank Buters and Sai Vinjanampathy.
[1] D. Rugar and P. Grütter, Mechanical Parametric Amplification and Thermomechanical Noise Squeezing, Phys. Rev. Lett. 67, 699 (1991).

[2] F. DiFilippo, V. Natarajan, K. R. Boyce, and D. E. Pritchard, Classical Amplitude Squeezing for Precision Measurements, Phys. Rev. Lett. 68, 2859 (1992).

[3] V. B. Braginsky, Y. I. Vorontsov, and K. S. Thorne, Quantum nondemolition measurements, Science 209, 547 (1980).

[4] A. Clerk, F. Marquardt, and K. Jacobs, Back-action evasion and squeezing of a mechanical resonator using a cavity detector, New J. Phys. 10, 095010 (2008).

[5] M. R. Vanner et al., Pulsed quantum optomechanics, Proc. Natl. Acad. Sci. USA 108, 16182 (2011).

[6] A. Szorkovszky, A. C. Doherty, G. I. Harris, and W. P. Bowen, Mechanical Squeezing via Parametric Amplification and Weak Measurement, Phys. Rev. Lett. 107, 213603 (2011).
[7] X. You, Z. Li, and Y. Li, Strong quantum squeezing of mechanical resonator via parametric amplification and coherent feedback, Phys. Rev. A 96, 063811 (2017).

[8] J. Hertzberg et al., Back-action-evading measurements of nanomechanical motion, Nat. Phys. 6, 213 (2010).

[9] J. Suh et al., Mechanically detecting and avoiding the quantum fluctuations of a microwave field, Science 344, 1262 (2014).

[10] E. E. Wollman et al., Quantum squeezing of motion in a mechanical resonator, Science 349, 952 (2015).

[11] F. Lecocq, J. B. Clark, R. W. Simmonds, J. Aumentado, and J. D. Teufel, Quantum nondemolition measurement of a nonclassical state of a massive object, Phys. Rev. X 5, 041037 (2015).

[12] J.-M. Pirkkalainen, E. Damskägg, M. Brandt, F. Massel, and M. A. Sillanpää, Squeezing of Quantum Noise of Motion in a Micromechanical Resonator, Phys. Rev. Lett. 115, 243601 (2015). 
[13] C. U. Lei, A. J. Weinstein, J. Suh, E. E. Wollman, A. Kronwald, F. Marquardt, A. A. Clerk, and K. C. Schwab, Quantum Nondemolition Measurement of a Quantum Squeezed State beyond the 3 db Limit, Phys. Rev. Lett. 117, 100801 (2016).

[14] T. Briant, P.-F. Cohadon, A. Heidmann, and M. Pinard, Optomechanical control of mirror motion at the attometer level, in Proceedings of the 2003 European Quantum Electronics Conference, EQEC 2003 (IEEE, Piscataway, NJ, 2003), p. 327.

[15] A. Vinante and P. Falferi, Feedback-Enhanced Parametric Squeezing of Mechanical Motion, Phys. Rev. Lett. 111, 207203 (2013).

[16] A. Szorkovszky, G. A. Brawley, A. C. Doherty, and W. P. Bowen, Strong Thermomechanical Squeezing via Weak Measurement, Phys. Rev. Lett. 110, 184301 (2013).

[17] M. Poot, K. Y. Fong, and H. X. Tang, Classical non-Gaussian state preparation through squeezing in an optoelectromechanical resonator, Phys. Rev. A 90, 063809 (2014).

[18] M. Poot, K. Fong, and H. Tang, Deep feedback-stabilized parametric squeezing in an opto-electromechanical system, New J. Phys. 17, 043056 (2015).

[19] A. Pontin, M. Bonaldi, A. Borrielli, F. S. Cataliotti, F. Marino, G. A. Prodi, E. Serra, and F. Marin, Squeezing a Thermal Mechanical Oscillator by Stabilized Parametric Effect on the Optical Spring, Phys. Rev. Lett. 112, 023601 (2014).

[20] M. Rashid, T. Tufarelli, J. Bateman, J. Vovrosh, D. Hempston, M. S. Kim, and H. Ulbricht, Experimental Realization of a Thermal Squeezed State of Levitated Optomechanics, Phys. Rev. Lett. 117, 273601 (2016).

[21] M. Vanner, J. Hofer, G. Cole, and M. Aspelmeyer, Coolingby-measurement and mechanical state tomography via pulsed optomechanics, Nat. Commun. 4, 2295 (2013).

[22] I. Mahboob, H. Okamoto, K. Onomitsu, and H. Yamaguchi, Two-Mode Thermal-Noise Squeezing in an Electromechanical Resonator, Phys. Rev. Lett. 113, 167203 (2014).

[23] Y. S. Patil, S. Chakram, L. Chang, and M. Vengalattore, Thermomechanical Two-Mode Squeezing in an Ultrahighq Membrane Resonator, Phys. Rev. Lett. 115, 017202 (2015).

[24] A. Pontin, M. Bonaldi, A. Borrielli, L. Marconi, F. Marino, G. Pandraud, G. A. Prodi, P. M. Sarro, E. Serra, and F. Marin, Dynamical Two-Mode Squeezing of Thermal Fluctuations in a Cavity Optomechanical System, Phys. Rev. Lett. 116, 103601 (2016).
[25] C. Ockeloen-Korppi et al., Stabilized entanglement of massive mechanical oscillators, Nature (London) 556, 478 (2018).

[26] B. S. Sheard, M. B. Gray, C. M. Mow-Lowry, D. E. McClelland, and S. E. Whitcomb, Observation and characterization of an optical spring, Phys. Rev. A 69, 051801 (2004).

[27] M. Aspelmeyer, T. J. Kippenberg, and F. Marquardt, Cavity optomechanics, Rev. Mod. Phys. 86, 1391 (2014).

[28] A. Jayich et al., Dispersive optomechanics: A membrane inside a cavity, New J. Phys. 10, 095008 (2008).

[29] J. Thompson et al., Strong dispersive coupling of a high-finesse cavity to a micromechanical membrane, Nature (London) 452, 72 (2008).

[30] E. D. Black, An introduction to Pound-Drever-Hall laser frequency stabilization, Am. J. Phys. 69, 79 (2001).

[31] F. M. Buters et al., High- $q$ nested resonator in an actively stabilized optomechanical cavity, Appl. Phys. Lett. 110, 104104 (2017).

[32] M. J. Weaver et al., Coherent optomechanical state transfer between disparate mechanical resonators, Nat. Commun. 8, 824 (2017).

[33] S. A. McGee, D. Meiser, C. A. Regal, K. W. Lehnert, and M. J. Holland, Mechanical resonators for storage and transfer of electrical and optical quantum states, Phys. Rev. A 87, 053818 (2013).

[34] W. Marshall, C. Simon, R. Penrose, and D. Bouwmeester, Towards Quantum Superpositions of a Mirror, Phys. Rev. Lett. 91, 130401 (2003).

[35] M. Weaver, D. Newsom, F. Luna, W. Löffler, and D. Bouwmeester, Phonon interferometry for measuring quantum decoherence, Phys. Rev. A 97, 063832 (2018).

[36] S. Sonar, M. Hajdušek, M. Mukherjee, R. Fazio, V. Vedral, S. Vinjanampathy, and L.-C. Kwek, Squeezing Enhances Quantum Synchronization, Phys. Rev. Lett. 120, 163601 (2018).

[37] A. Nunnenkamp, V. Sudhir, A. K. Feofanov, A. Roulet, and T. J. Kippenberg, Quantum-Limited Amplification and Parametric Instability in the Reversed Dissipation Regime of Cavity Optomechanics, Phys. Rev. Lett. 113, 023604 (2014).

[38] Y. Tsaturyan, A. Barg, E. S. Polzik, and A. Schliesser, Ultracoherent nanomechanical resonators via soft clamping and dissipation dilution, Nat. Nanotechnol. 12, 776 (2017).

[39] M. Rossi, D. Mason, J. Chen, Y. Tsaturyan, and A. Schliesser, Measurement-based quantum control of mechanical motion, arXiv:1805.05087. 